\title{
Research on Mandatory Lane-Changing Behavior in Highway Weaving Sections
}

\author{
Wei Hao $\mathbb{D}^{1},{ }^{1}$ Zhaolei Zhang, ${ }^{1}$ Zhibo Gao $\mathbb{D},{ }^{2}$ Kefu Yi $\left(D^{3}{ }^{3}\right.$ Li Liu, ${ }^{3}$ and Jie Wang ${ }^{1}$ \\ ${ }^{1}$ Hunan Key Laboratory of Smart Roadway and Cooperative Vehicle-Infrastructure Systems, \\ Changsha University of Science \&Technology, Changsha 410205, China \\ ${ }^{2}$ The Key Laboratory of Road and Traffic Engineering, Ministry of Education, Tongji University, 4800 Cao'an Road, \\ Shanghai 201804, China \\ ${ }^{3}$ School of Automotive and Mechanical Engineering, Changsha University of Science \& Technology, Changsha 410076, China
}

Correspondence should be addressed to Wei Hao; haowei@csust.edu.cn, Zhibo Gao; gaozhibo@tongji.edu.cn, and Kefu Yi; corfyi@ csust.edu.cn

Received 2 January 2020; Revised 25 June 2020; Accepted 29 June 2020; Published 23 July 2020

Academic Editor: Young-Ji Byon

Copyright (c) 2020 Wei Hao et al. This is an open access article distributed under the Creative Commons Attribution License, which permits unrestricted use, distribution, and reproduction in any medium, provided the original work is properly cited.

As the accident-prone sections and bottlenecks, highway weaving sections will become more complicated when it comes to the mixed-traffic environments with connected and automated vehicles (CAVs) and human-driven vehicles (HVs). In order to make CAVs accurately identify the driving behavior of manual-human vehicles to avoid traffic accidents caused by lane changing, it is necessary to analyze the characteristics of the mandatory lane-changing (MCL) process in the weaving area. An analytical MCL method based on the driver's psychological characteristics is proposed in this study. Firstly, the driver's MLC pressure concept was proposed by leading in the distance of the off-ramp. Then, the lane-changing intention was quantified by considering the driver's MLC pressure and tendentiousness. Finally, based on the lane-changing intention and the headway distribution of the target lane, an MLC positions probability density model was proposed to describe the distribution characteristics of the lane-changing position. Through the NGSIM data verification, the lane-changing analysis models can objectively describe the vehicle lanechanging characteristics in the actual scenarios. Compared with the traditional lane-changing model, the proposed models are more interpretable and in line with the driving intention. The results show significant improvements in the lane-changing safe recognition of CAVs in heterogeneous traffic flow (both CAVs and HVs) in the future.

\section{Introduction}

As one of the basic driving behaviors, lane-changing manoeuver directly affects the fluency and safety of traffic flow. Compared with car-following, the lane-changing process is more complicated and dangerous. Previous studies have shown that lane changing is a key factor causing highway accidents [1-7]. As CAVs enter the road, highway weaving areas will become more chaotic and dangerous. $\mathrm{CAV}$, as a passive party, needs to change its operating state in real time according to the operating state of manualhuman vehicles to avoid collisions. Therefore, it is crucial to study the lane-changing characteristics of manual-human vehicles to avoid vehicle collisions. However, most researches mainly use statistical models and probability models to analyze various factors influencing traffic accidents in a fixed scenario, lacking the analysis of traffic flow theory, which is of low extensibility [8]. Based on the above research foundation, this paper will use the traffic flow theory and driver characteristics to analyze the vehicles' lane-changing behavior in expressway weaving area.

The lane-changing manoeuver is divided into discretionary lane change (DLC) and mandatory lane change (MLC) according to its motivation [9, 10]. MLC is the necessary lane-changing behavior to achieve certain demands; DLC is an unnecessary lane change behavior. It can be seen that the MLC is an aggressive and necessary manoeuver, which has a great influence on the traffic flow. As an important part of the highway, the weaving section undertakes the merging and the diverging of the traffic flow. 
In weaving section, all lane-changing manoeuvers belong to MLC (driving into the highway; leaving the highway), which are the manoeuver that must be completed in time; otherwise, it will affect the operation of the highway segment. So the weaving section becomes the bottleneck of the highway $[3-5,11,12]$.

In the past research, in order to simplify and facilitate the modeling, it is considered that the lane-changing process is only affected by the traffic conditions (the gap of the target lane, the traffic capacity, and the speed, etc.). Sun and Elefteriadou [13] found that the MLC process is also closely related to the driver's psychological characteristics (such as aggressiveness, vigilance, and lane-changing pressure) through data investigation. However, the existing models have not fully analyzed the influence of the driver's psychological characteristics on the lane-changing process [13].

To address this challenge, the concept of lane-changing pressure is introduced to describing the drivers' pressure fluctuation in MLC. The MLC pressure is quantified by the distance between the vehicle and the off-ramp. Then, using the lane-changing pressure as an indicator, this paper proposed an MLC intention model and a gap acceptance model to analyze the driver's intention to change lanes. The proposed model is based on the driver's intention and inclination, which overcomes the defect of traditional models only using traffic conditions as a fixed indicator. Finally, combining the target lane headway distribution and MLC intention, a lane change probability density model is proposed to describe the lane-changing characteristics in the weaving area.

The rest of the paper is structured as follows: Section 2 reviews previous literature on mandatory lane-changing models. Section 3 analyzes the characteristics of MLC and proposes that the MLC is a continuous behavior. Section 4 constructs an MLC positions probability density function. Section 5 uses NGSIM data to verify the proposed theory and models. Finally, the conclusions and future work are presented in Section 6.

\section{Literature Review}

At present, the lane-changing decision-making models mainly include rule-based models [14-19], discrete models based on utility theory $[9,10,20,21]$, and artificial intelligence models [22-28].

The main idea of the rule-based model is to formulate different driving rules according to different driving environments. The advantages of rule-based models are traceability and simple implementation for specific scenarios. The drivers choose whether to change lanes by some simple rules. However, for complicated traffic conditions, a rule-based model can require a substantial work in order to be extended into more general scenarios. The most representative rulebased model is Gipps' lane-changing model [14]. Gipps believes that a driver's lane-changing decision is the result of three problems: (1) Is it possible to change lanes? (2) Is it necessary to change lanes? (3) And is it desirable to change lanes? After Gipps' pioneering work, many people have expanded the lane-changing decision-making model, such as CORSIM Model [29], ARTEMiS Model [12], Cellular Automata Model [30], and Game Theory Model [31]. By extending Gipps' model to the highway, Yang and Koutsopoulos [19] invented a microscopic traffic simulator (MITSIM) that includes a car-following model and a lanechanging model. They classify lane changing as mandatory or discretionary and model the lane-changing decisionmaking as a continuous four-step process: (1) decide to consider the LC, (2) select the target lane, (3) find acceptable gaps, (4) and implement the changes. The gap acceptance algorithm receives and checks for gaps in the target lane to perform the required lane change. Although the rule-based modeling framework in Yang et al. is similar to the Gipps' model (1986), one of the distinguishing features of their model is that, instead of considering the lane-changing decision-making as a deterministic process, it introduces the lane-changing probability to build the model.

The main idea of the discrete model is to use the utility function to evaluate the driving gain of each lane. Discrete models based on utility functions have the advantage of allowing evaluation of multiple decision criteria by combined weighting and can thus more easily be extended to complex scenarios. However, a large number of different weighting parameters can result in time-consuming parameter tuning and tractability difficulties, and vehicle lane change is a continuous process. The first discrete-choice model based on the utility function was proposed by Ahmed et al. $[10,11]$ and further refined by Toledo et al. $[20,32]$. Ahmed et al. [9, 10] used the gap between the vehicles as the main influencing factor of the utility function, using the utility function to simulate the impact of the gap on the driver. Then, through the field data, the binomial logit model is used to calibrate the weighting parameters of the utility function. Ahmed's model divides the lane change type into MLC and DLC but does not explain the driver's choice of both sides. For a clearer understanding of the type of vehicle lane change, based on the basis of the Ahmed model, Toledo et al. [20, 32] proposed a probabilistic lane-changing decision model to describe the relationship between MLC and DLC. The relationship is captured by considering two types of lane changes in a single utility function, and a discrete-choice framework is chosen to simulate the strategy and operation of the driver's lane change decision. Most of the above studies are modeled on vehicle trajectory data, even though driver characteristics have a significant impact on all aspects of the lane process. But driver characteristics are not considered because driver's feature data extraction is difficult and the workload is huge, so most lane change decision models lack them. To explicitly incorporate the impact of driver characteristics, Sun and Elefteriadou [13] conducted a survey to determine and understand the driver's driving behavior in various lane change scenarios. The study reveals the types, causes, and main factors of each driver type in the lane-changing decision process and the links between them.

The artificial intelligence lane-changing decision methods use a computer to simulate the driver's thinking 
and actions during the driving process and judge the environment to determine whether it is necessary to change lanes, whether it is necessary to change lanes, and the choice of target lanes. The artificial intelligence models impose some artificial intelligence algorithms, such as fuzzy logic [22], artificial neural networks [23], and Bayesian classification [24, 25], to explore the potential determinants of driver lane change behavior. The artificial intelligence models are completely data-driven and do not have any physical meaning parameters; it is inconvenient to analyze the performance of the method and the scenario expansion and does not consider the driver's psychological factors.

Through a review of the lane-changing models, it is found that the existing lane-changing models have not fully considered the driver's influence on the lane changing. However, these features are important for accurately describing the lane-changing behavior, and the relevant explanatory variables should be included in the future lanechanging model. In this paper, a new research idea based on lane-changing pressure is proposed to express the characteristics of the MCL in highway weaving section, and an MCL model is established based on the driver's driving characteristics. The traditional methods only consider the influence of the traffic flow characteristics of the target lane on the lane change. Considering the driver's psychology of being forced to change lanes, this paper introduces the driver's pressure to lane change (which is reflected by the distance between the vehicle and the off-ramp) into the MCL model, which is supposed to better describe the actual traffic situation.

\section{Mandatory Lane Change Behavior}

MLC is a necessary lane-changing behavior to achieve a certain demand, such as merging and diverging. DCL is an unessential lane-changing behavior, usually to achieve expectations speed or keep a certain distance from the car in front $[9,10]$. Therefore, compared with the DCL, the drivers who have MLC demand will improve aggressiveness, and it is easy to cause traffic accidents. For this issue, this paper studies the MLC scenarios of vehicles leaving the highway, as shown in Figure 1. When the vehicle has the demand leaving the highway, it will choose whether to change lanes to the right lane according to the current driving environment, then enter the weaving section, and finally enter the off-ramp. The MLC process is a continuous process and is divided into four phases: (1) determining the target lane, (2) generating the intention to change lanes, (3) finding acceptable gaps, and (4) executing lane changing. The lane-changing decision process is shown in Figure 2. The MLC is a complicated process involving road conditions (total traffic capacity, vehicle arrival rate of on-ramp and off-ramp, and auxiliary lane layout), driver characteristics, and so on. Therefore, it is difficult to quantify various factors. After introducing the concept of driver's lane-changing pressure, this paper will systematically study the various stages of the MLC process.

\section{MLC Models}

4.1. MLC Pressure. MLC pressure refers to the pressure generated by the drivers who havse lane-changing demand before the last lane-changing node (LLCN). It is easy to know that the closer the driver is to the off-ramp, the stronger the desire to change lanes is generated. So, assume that the driver's MLC pressure is quantified by the available parameter of distance in this paper. The quantitative models were proposed as follows:

$$
f= \begin{cases}{\left[\omega\left(\frac{x_{n}-d_{i}}{S}\right)\right]^{-\alpha},} & d_{i} \leq x_{n} \leq S, \\ 0, & x_{n}>S,\end{cases}
$$

where $f$ denotes the lane-changing pressure value; $x_{n}$ denotes the distance of the vehicle $n$ from the off-ramp; $d_{i}$ denotes the distance of the LLCN of the lane $i$ from the offramp; $S$ denotes the length of the weaving. While the vehicle does not enter the weaving $\left(x_{n}>S\right)$, the drivers do not have MLC pressure; that is, $f=0$. While $x_{n}<S$, the pressure that the driver starts to generate, the closer the distance to the offramp, the greater the lane change pressure. While the vehicle reaches the $\operatorname{LLCN}\left(x_{n}=d_{i}\right)$, the MLC pressure is $\infty$, and the vehicle must change lanes. $\alpha \in N^{*}$.

The formula for calculating $\omega$ is as follows:

$$
\omega=\beta_{0}+\beta_{1} N+\beta_{2} \lambda_{1}+\beta_{3} \lambda_{2},
$$

where $\beta_{0}, \beta_{1}, \beta_{2}$, and $\beta_{3}$ denote coefficient of each parameter, respectively; $N$ denotes the number of lanes that need to be crossed; $\lambda_{1}$ denotes the average arrival rate of vehicles on the segment at time unit; $\lambda_{2}$ denotes the average arrival rate of the on-ramp vehicles at time unit.

The MLC pressure model is helpful to analyze the influence of the driver's psychological factors on the lane-changing behavior. Secondly, quantifying the driver's pressure is helpful to promote the identification and behavior prediction of the MHV characteristics on the heterogeneous traffic flow in the future and reduce the conflict between the CAVs and the MHV. Some characteristic parameters of drivers (gender, age, occupation, etc.) can also be added into the model.

4.2. MLC Intention. Yang and Koutsopoulos [19] proposed that mandatory lane changing occurs when drivers have to change lanes in order to

(a) connect the link on their path

(b) bypass a lane blockage downstream

(c) avoid entering a restricted use lane

(d) respond to lane use sign or speed limit sign

The MCL studied by Yang et al. are limited to (b) and (c). In addition, the intention to change lanes in the weaving area belongs to (a); drivers tend to change lanes early to avoid the lane-changing pressure. That is, the lower the pressure, the stronger the driver's intention to change lanes. Based on the MLC model, a new MLC intention model is proposed: 


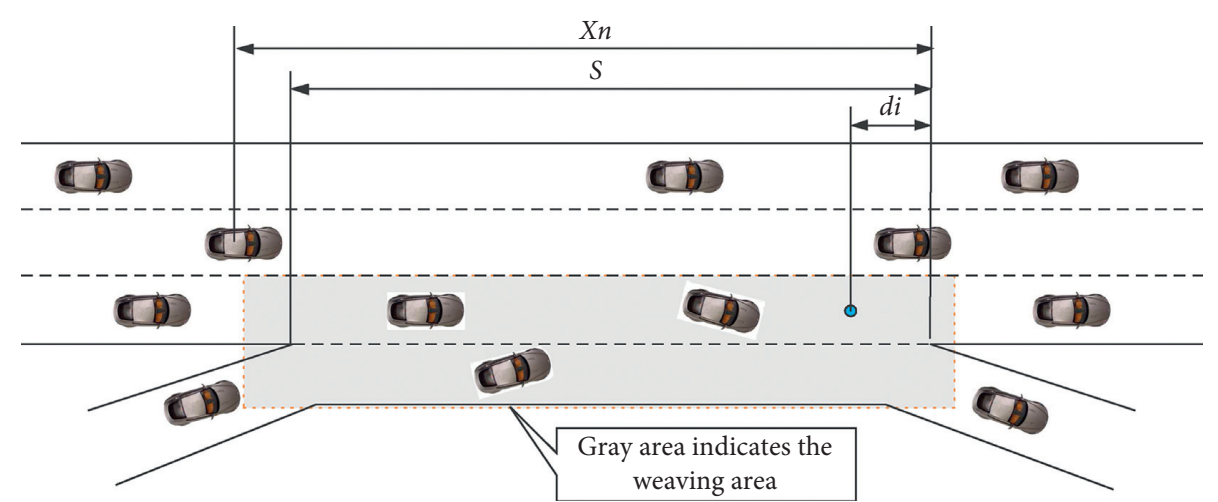

FIGURE 1: Highway weaving area.

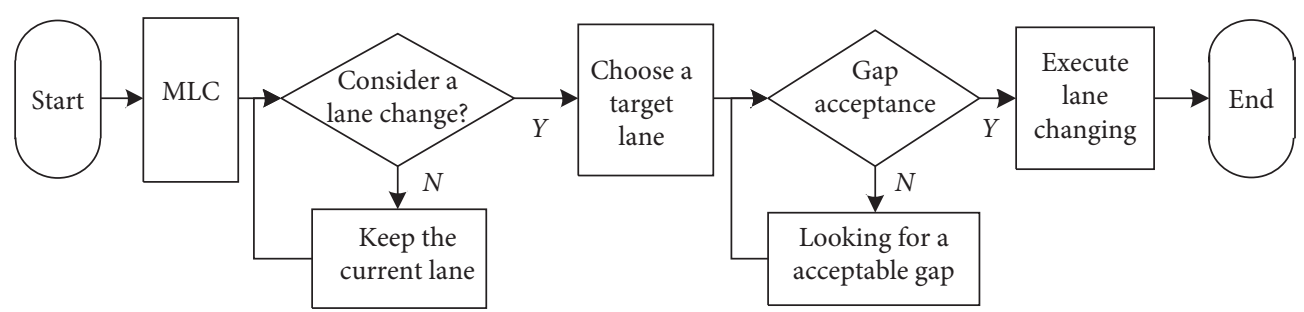

FIGURE 2: Lane-changing process.

$$
\begin{aligned}
& \varphi\left(x_{n} / t_{x_{n}} \geq t_{x_{n}-\min }\right)=\exp \left(-f\left(x_{n}\right)^{-1}\right) \\
&=\exp \left[-\frac{\left[\left(\beta_{0}+\beta_{1} N+\beta_{2} \lambda_{1}+\beta_{3} \lambda_{2}\right)\left(x_{n}-d_{i}\right)\right]^{\alpha}}{S^{\alpha}}\right], \\
& d_{i} \leq x_{n} \leq S,
\end{aligned}
$$

where $\varphi\left(x_{n} / t_{x_{n}} \geq t_{x_{n-\min }}\right)$ denotes the intention generated of the vehicle $n$ at a distance of $x$ meters from off-ramp. $t_{x_{n}} \geq T_{x_{n-} \text { min }}$ denotes that there is a traversable gap in the target lane, which will analyze the determination of the traversable gap in the next section. The MLC intention model describes the intensity of the MLC intention at different location. The model is helpful to analyze the driving behavior characteristics in the weaving section and also obtains some driving potential preferences of the driver.

4.3. Gap Acceptance. The lane-changing decision is not only determined by the driver's personal driving-preference but also closely related to the driving environment. Only when there is an acceptable gap in the target lane, the vehicles can enter into the target lane. Therefore, the headway of the target lane is the key factor for the successful implementation of lane changing. Erlang distribution is a more general distribution model of traffic characteristics such as headway and speed. According to the change of parameter " $k$ " in the distribution function, there are different distribution functions. The Erlang probability density function is as follows (as shown in Figure 3):

$$
g(t)=\lambda e^{-\lambda t} \frac{(\lambda t)^{k-1}}{(k-1) !}, \quad k=1,2,3, \ldots
$$

When $k=1$, the Erlang distribution is equal to the negative exponential distribution; when $k=\infty$, a steady headway distance is produced. This shows that the parameter $k$ in the Erlang distribution can reflect the conditions of various traffic flows between the free traffic flow and the crowded traffic flow. As the value of $k$ increases, the more crowded the traffic is, the more crowded the traffic flow becomes. So that, it is difficult for drivers to drive freely. Therefore, the value of $k$ is a rough representation of the degree of nonrandomness, and the degree of nonrandomness increases as the value of $k$ increases.

The value of $k$ is calculated as follows:

$$
\begin{aligned}
k & =\frac{m^{2}}{S^{2}}, \\
m & =\lambda_{1} t=\frac{\sum_{j=1}^{g} k_{j} f_{j}}{\sum_{j=1}^{g} f_{i}}=\frac{\sum_{j=1}^{g} k_{j} f_{j}}{N}, \\
S^{2} & =\frac{1}{N-1} \sum_{i=1}^{N}\left(k_{i}-m\right)^{2}=\frac{1}{N-1} \sum_{i=1}^{g}\left(k_{i}-m\right)^{2} f_{i},
\end{aligned}
$$

where $m$ denotes the vehicle arrival rate during time $t ; S^{2}$ denotes the variance; $t$ denotes the duration $(s)$ or distance $(m)$ of each count interval; $g$ denotes the number of the groups; $f_{i}$ denotes the frequency of the number of vehicles arriving is $k_{i}$ in $t ; k_{i}$ denotes the number of vehicles arriving in $t$; $N$ denotes the total number of observation intervals; $K$ is an integer.

Previous studies [12, 32] assumed that the safe crossing gap was a fixed value or followed a normal distribution. However, due to the fact that lane changing is mandatory in the weaving area, the closer the vehicle was to the LLCN, the 


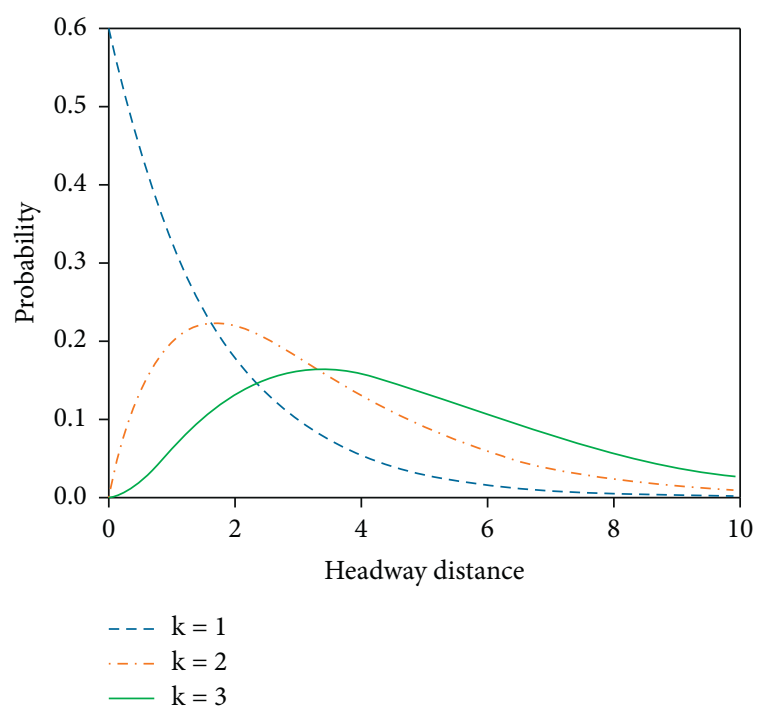

Figure 3: Erlang distribution.

greater the driver's desire to enter the target lane was. As a result, it is assumed that the minimum acceptable gap is proportional to the distance and proposes a minimum acceptable gap model:

$$
t_{x_{n-\min }}=\frac{t_{d}}{S}\left(S-x_{n}\right)
$$

where $t_{x_{n-} \text { min }}$ is minimum acceptable merging headway chosen by the vehicle $n . t_{d}$ is minimum acceptable merging headway expected by drivers in general driving environment.

So, the probability of acceptable gap in the target lane is as follows:

$$
\begin{aligned}
G\left(h \geq t_{x_{n}-\min }\right) & =\int_{t_{x_{n}}}^{\infty} \lambda e^{-\lambda t_{x}} \frac{\left(\lambda t_{x_{n}}\right)^{k-1}}{(k-1) !} \\
& =\sum_{i=0}^{i}\left(\lambda k t_{x_{n-} \min }\right) \frac{i e^{-\lambda k t_{x_{n}}}}{i !} .
\end{aligned}
$$

The selection of manual-human vehicles' lane-changing gap is dynamic, which has great interference to CAVs trajectory planning. If CAVs are blindly conservative or aggressive, it is easy to cause vehicle collisions. Therefore, the acceptable gap acceptance model proposed in this paper can provide an important technical means for MCL identification of CAVs, which provides a guarantee for traffic safety in mixed-traffic flows.

4.4. MLC Execution. Two conditions should be met for the successful implementation of MLC: the driver has the MLC intention and the target lane has an acceptable gap. When both conditions are met, the vehicle can successfully change lanes to the target lane. Suppose the driver has the intention to change lanes which is event $A$, and there is the acceptable gap in the target lane which is event $B$. According to the lane change intention model and headway distribution model proposed previously, the MLC probability density function can be obtained:

$$
\begin{aligned}
P\left(x_{n}\right)= & P(A B)=P(A) P(B), \\
P\left(x_{n}\right)= & \exp \left[\frac{-\left(x_{n}-d_{i}\right)^{\alpha}}{S^{\alpha}\left(\beta_{0}+\beta_{1} N+\beta_{2} \lambda_{1}+\beta_{3} \lambda_{2}\right)^{-\alpha}}\right] \\
& \cdot \int_{\left(t_{d} / S\right) x_{n}}^{\infty} \lambda e^{-\lambda t_{x}} \frac{\left(\lambda\left(t_{d} / S\right) x_{n}\right)^{k-1}}{(k-1) !},
\end{aligned}
$$

where $P\left(x_{n}\right)$ is the probability density function of MLC in the weaving area. This model can predict the lane-changing behavior for advanced driver assistance system (ADAS) to avoid crash and trajectory planning $[27,28]$.

\section{Case Study and Models Verification}

5.1. Scenarios and Data Description. The NGSIM trajectory data is obtained by the US Federal Highway Administration (FHWA) for the purpose of conducting the "Next Generation Simulation" program [33]. The data is collected by the high-altitude camera to capture the vehicle's driving process, and then the video processing software is used to restore the precise position of each vehicle in the study section at 10 frames per frame or 15 frames per second. This paper uses the data of the US-101 highway section in NGSIM. The length of the detected section is 640 meters and there are five lanes. In addition, it also includes a couple of ramps and an auxiliary lane. The effective data is obtained by processing the trajectory data, as shown in Table 1 . The road segment diagram is shown in Figure 4.

It can be seen from the figure that the segment has a weaving section and the collected trajectory data is complete. 157 cars which have finished MLC were extracted from the data.

\subsection{MLC Analysis and Models Verification}

5.2.1. MLC Intention Model. In order to accurately capture the driver's intention to change lanes and to verify the reliability of the MLC intention model proposed in this paper, we extracted some trajectory in free flow. That is, there are no vehicles in auxiliary lane (lane 6) during this period. As shown in Figure 5, in the absence of other vehicle interferences, lane-changing positions are mainly concentrated at 140-200 meters from the downstream. There are few vehicles that choose to change lanes at the end of lane 5 . The reason for this phenomenon is that drivers with MLC demand tend to enter lane 6 as early as possible to relieve pressure. While $\lambda_{2}=0$, the MLC intention model is as follows:

$$
\varphi\left(x_{n} \mid \lambda_{2}=0, i=1\right)=\exp \left[\frac{-\left(x_{n}-d_{i}\right)^{\alpha}}{S^{\alpha}\left(\beta_{0}+\beta_{1} N+\beta_{2} \lambda_{1}\right)^{-\alpha}}\right] .
$$

As shown in Figure 6, the curve represents the relationship between $\varphi$ and $x_{n}$, and the histogram represents the 
TABLE 1: Detecting road parameters.

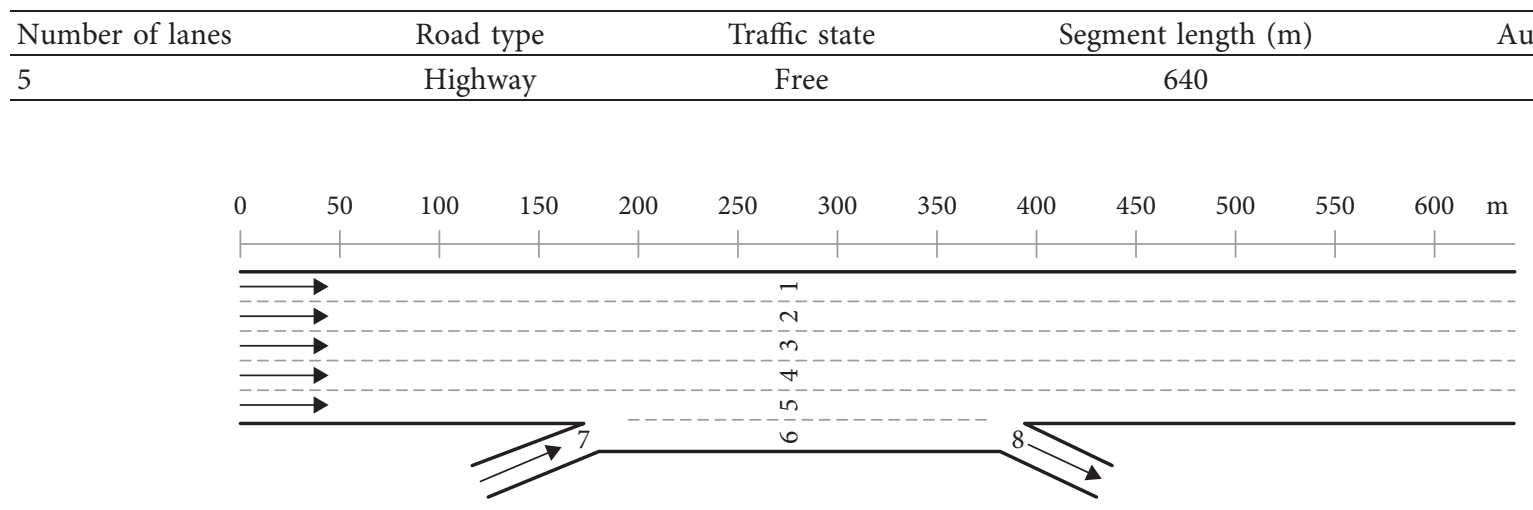

FIgURE 4: Detecting road geometry.

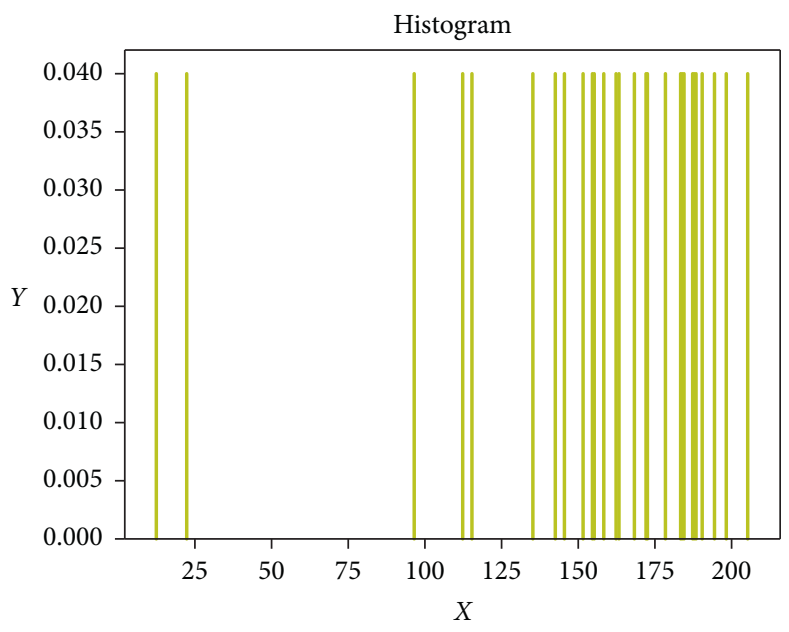

FIgURE 5: Lane-changing points distribution.

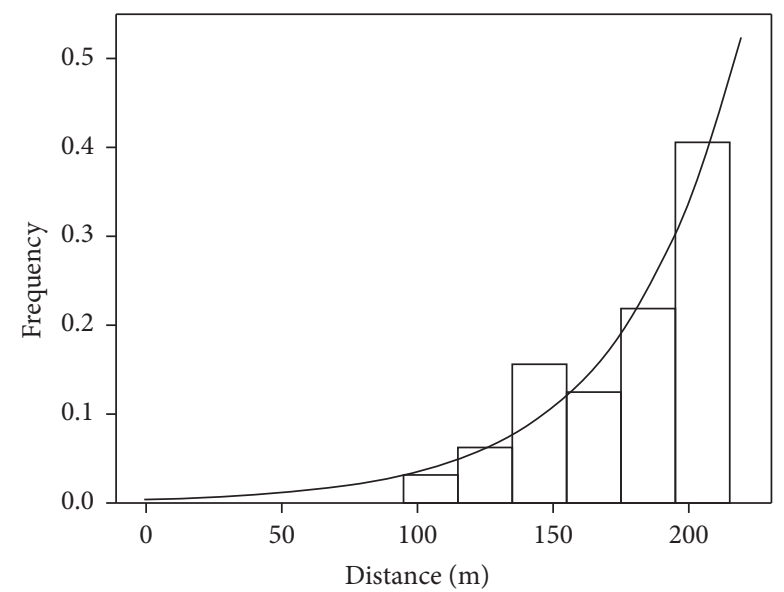

FIGURE 6: Lane-changing willingness fitting.

frequency at which all vehicles change lanes at $x_{n}$ during the detection period. The fit of the model proposed is $92.3 \%$, which can more accurately describe the intention of the driver to change lanes.
5.2.2. Gap Acceptance Model. When there is no vehicle in front of the subject vehicle within the detection section, the default ID of the vehicle in front is " 0 ," and the headway is also "0." Although the headway is zero in data, but the actual 
headway is large. To this end, we divide the headway into two parts. The first part is that there is a certain headway, and the other part is "0." Therefore, the driver's acceptable headway is divided into two parts $t=0$ or $t \geq t_{a}$ :

$$
t= \begin{cases}\text { Refuse changing lanes, } & 0<t<t_{x_{n-} \min }, \\ \text { Accept changing lanes, } & t=0 \text { or } t \geq t_{x_{n-} \text { min }},\end{cases}
$$

where $t$ denotes headway.

This paper uses the Erlang distribution to fit the lane headway of lane 6, as shown in Figure 7 . It is found that the frequency of the headway is zero, accounting for $43 \%$, which indicates that the weaving zone is in good operating condition. When $k=2$, the Erlang distribution function fits better to the headway of lane 6 (Figure 8).

The driver's acceptable gap is related to the driver's own driving style and is also related to the distance from onramp. The vehicle headway $\left(t_{x_{-} \max }\right)$-distance $\left(x_{n}\right)$ scatter plot is drawn by collecting the headway data at the time of the vehicles change lanes. Vehicles mainly change lanes within 150-200 meters. As the distance decreases, the range of the headway is also reduced. They conform to the following relationship:

$$
t_{\min }<t_{a} \leq t_{x_{-} \max }=S \cdot e^{\left(-2 / x_{n}\right)} .
$$

5.2.3. MLC Positions Distribution. In this paper, the local coordinates of the MLC lane-changing positions are collected in weaving section of US-101 highway. The trajectory scatter plot (Figure 9) and heat map (Figure 10) of the lane change position are drawn according to the local coordinates. Lane 6 starts at 636.7 feet and ends at 1333.8 feet, respectively. As can be seen from the two figures, lane change points are mainly concentrated in the range of $650-750$ feet in the horizontal coordinate. Drivers tend to change lanes at the front of weaving area to lane 6 which can eliminate the pressure of lane change, instead of choosing lanes after measuring lane utility. This indicates that there is a trace to follow when the vehicle chooses to change lanes. And the rule of driver changing lanes can provide important guidance for CAVs lane-changing recognition to avoid collision.

According to the MCL probability density function proposed in this paper, the following formula can be obtained:

$$
\begin{aligned}
P\left(x_{n}\right)= & \varphi\left(x_{n} / t_{x_{n}} \geq T_{x_{-} \min }\right) \cdot G\left(h \geq t_{x}\right), \\
= & \left(1+\lambda_{2} \frac{t_{d}}{S} x_{n}\right) \exp \left[\frac{-\left(x_{n}-d\right)}{\beta_{0}+\beta_{1} N+\beta_{2} \lambda_{1}+\beta_{3} \lambda_{2}}-\frac{2 \lambda_{2} t_{d}}{S} x_{n}\right] \\
& +0.43 \cdot \exp \left[\frac{\left(x_{n}-d\right)}{\beta_{0}+\beta_{1} N+\beta_{2} \lambda_{1}}\right],
\end{aligned}
$$

where $\mathrm{P}\left(x_{n}\right)$ represents MLC probability density function and 0.43 in the model represents the proportion when the headway is zero, as shown in Figure 7. It can be seen from Figure 11 that the model proposed in this paper can

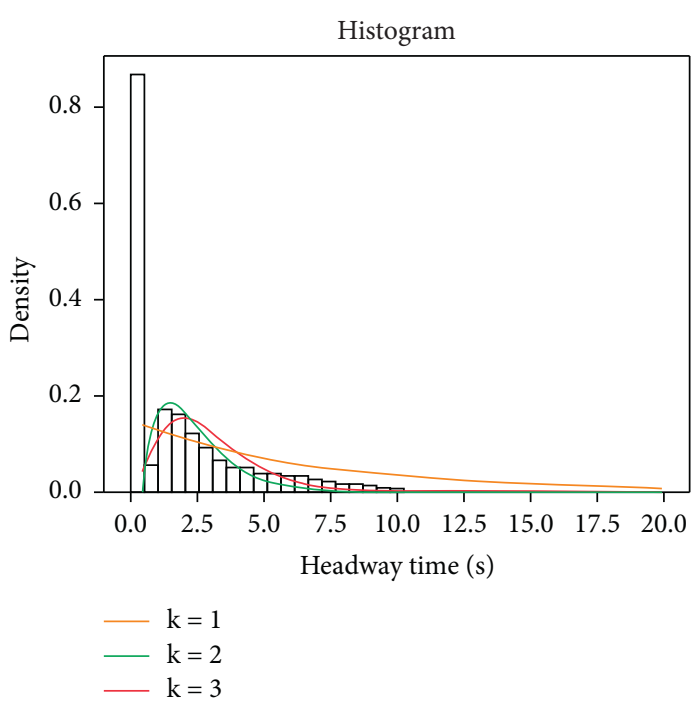

FIgURe 7: Headway distribution.

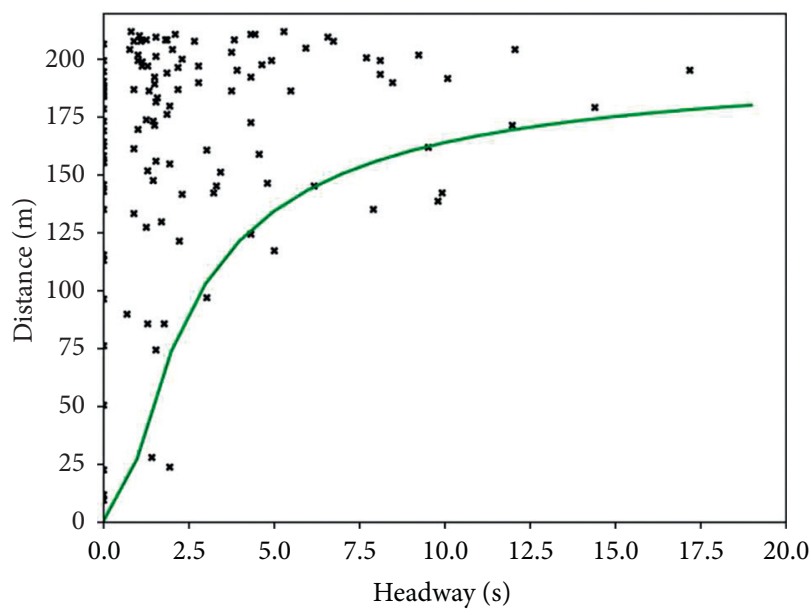

Figure 8: Headway of lane changing.

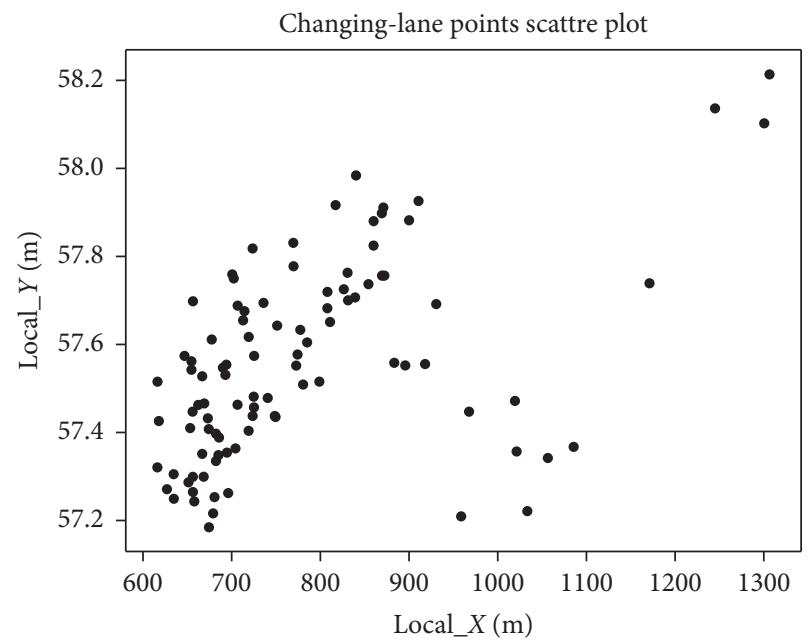

FIgUre 9: Lane-changing positions. 


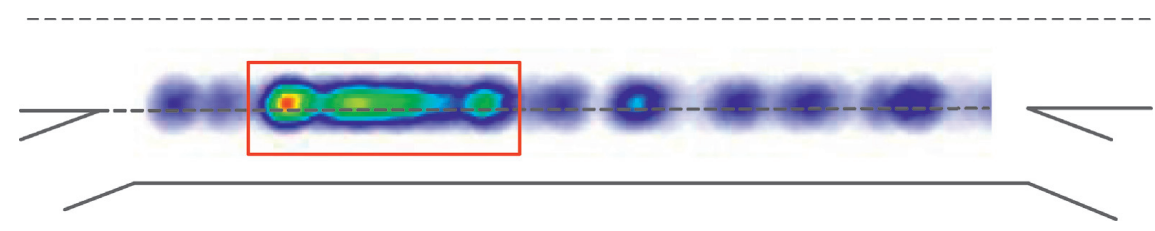

FIGURE 10: Lane-changing location heat map.

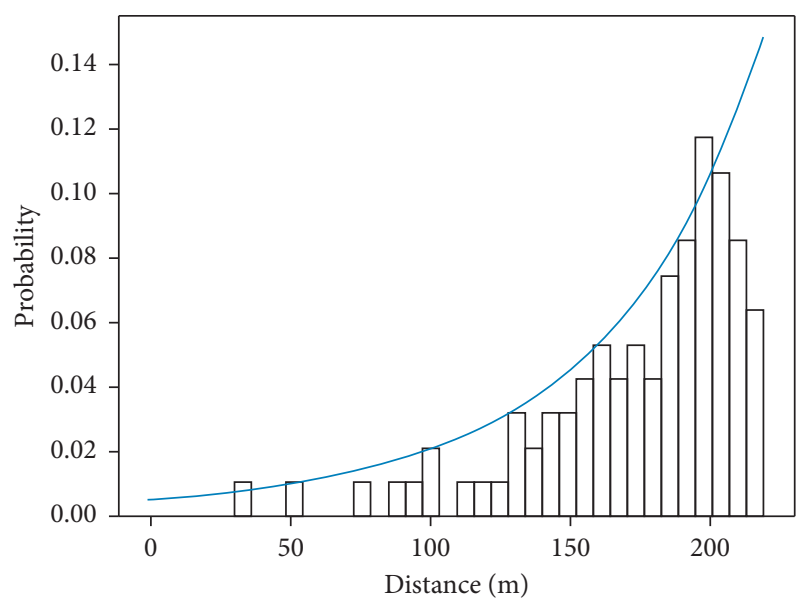

FIGURE 11: Histogram of lane-changing position.

accurately describe the drivers' selection trend of the lanechanging timing in the weaving section. But there is a difference in a part at $x_{n}>200$ meters for the reason that when establishing the MLC pressure model, this paper assumes that the pressure of the lane change is inversely proportional to the intention which makes the default optimal position of changing lanes be at front position of the auxiliary lane. However, when the pressure accumulates to a certain value, the MCL intention reaches the maximum value. How much pressure the driver generates is needed to be studied; the MCL intention will reach the peak.

\section{Conclusion}

Inspired by the principle of discretionary lane change models, this paper proposes a method based on driver's psychological pressure to analysis MLC. The main factor for driving the driver to DLC is the lanes' utility, and the main factor for driving the driver to MLC is the lane-changing pressure. Therefore, this paper proposes a new concept named lane-changing pressure to analyze the MLC stages. And obtaining the following research results, (1) the MLC pressure model is proposed by leading in the lane-changing pressure from the distance of the off-ramp. (2) Based on the MLC pressure, an MLC intention model is proposed, which describes the driver's preference for lane-changing positions in the weaving section. (3) By researching the driver's MLC intention and the probability of acceptable gaps, an MLC positions probability density function is proposed.

It is verified that the proposed models can objectively describe the characteristics of the lane-changing process in the weaving section by the NGSIM data. Compared with the traditional model, the proposed models explore the vehicle lane-changing process from the driver's level, which is more explanatory and expandable. It can provide the basis for CAVs vehicle to change lane recognition in heterogeneous traffic flow in the future.

\section{Data Availability}

The data used to support the findings of this study are available from the corresponding author upon request.

\section{Conflicts of Interest}

The authors declare that there are no conflicts of interest regarding the publication of this paper.

\section{Acknowledgments}

This work was supported by the Natural Science Foundation of China (nos.51808057, 71861023, and 61403047) and in a part supported by Hunan Provincial Natural Science Foundation of China (no. 2019JJ30026), the Young Elite Scientists Sponsorship Program by Hunan province of China (2018RS3074), Hunan Provincial Natural Science Foundation of China (2018JJ3553), Changsha Science and Technology Bureau Project (kq1801056), Open Fund of Hunan Key Laboratory of Smart Roadway and Cooperative Vehicle-Infrastructure Systems (kfj180702 and kfj190702), Innovation Team Project for Transportation Engineering in CSUST, and Ministry of Science Project with Montenegro $(3-2)$.

\section{References}

[1] Y. Ali, M. M. Haque, Z. Zheng, S. Washington, and M. Yildirimoglu, "A hazard-based duration model to quantify the impact of connected driving environment on safety during mandatory lane-changing," Transportation Research Part C: Emerging Technologies, vol. 106, pp. 113-131, 2019.

[2] H. Adeli and A. Karim, "Fuzzy-wavelet RBFNN model for freeway incident detection," Journal of Transportation Engineering, vol. 126, no. 6, pp. 464-471, 2000.

[3] H. Adeli and A. Samant, "An adaptive conjugate gradient neural network-wavelet model for traffic incident detection," Computer-Aided Civil and Infrastructure Engineering, vol. 15, no. 4, pp. 251-260, 2000.

[4] S. Ahn and M. J. Cassidy, "Freeway traffic oscillations and vehicle lane-change maneuvers," in Proceedings of the 17th International Symposium of Transportation and Traffic Theory, R. E. Allsop, M. G. H. Bell, and B. G. Heydecker, Eds., pp. 691-710pp. 691-, London, UK, July 2007. 
[5] A. Duret, S. Ahn, and C. Buisson, "Passing rates to measure relaxation and impact of lane-changing in congestion," Computer-Aided Civil and Infrastructure Engineering, vol. 26, no. 4, pp. 285-297, 2011.

[6] S. Ghosh-Dastidar and H. Adeli, "Wavelet-clustering-neural network model for freeway incident detection," ComputerAided Civil and Infrastructure Engineering, vol. 18, no. 5, pp. 325-338, 2003.

[7] Q. Zeng, W. Gu, X. Zhang, H. Wen, J. Lee, and W. Hao, "Analyzing freeway crash severity using a Bayesian spatial generalized ordered logit model with conditional autoregressive priors," Accident Analysis \& Prevention, vol. 127, pp. 87-95, 2019.

[8] M. Rahman, M. Chowdhury, Y. Xie, and Y. He, "Review of microscopic lane-changing models and future research opportunities," IEEE Transactions on Intelligent Transportation Systems, vol. 14, no. 4, pp. 1942-1956, 2013.

[9] K. I. Ahmed, Modeling Drivers' Acceleration and Lane Changing behavior, Massachusetts Institute of Technology, Cambridge, CA, USA, 1999.

[10] K. L. Ahmed, M. Ben-Akiva, H. Koutsopoulos, and R. G. Mishalani, "Models of freeway lane changing and gap acceptance behavior," in Transportation and traffic theory. Proceedings of the 13th International Symposium on Transportation and Traffic Theory, vol. 13, pp. 501-515, Lyon, France, July 1996.

[11] Q. Zeng, H. Wen, H. Huang, and M. Abdel-Aty, "A Bayesian spatial random parameters Tobit model for analyzing crash rates on roadway segments," Accident Analysis \& Prevention, vol. 100 , pp. 37-43, 2017.

[12] F. Chen, M. Song, and X. Ma, "Investigation on the injury severity of drivers in rear-end collisions between cars using a random parameters bivariate ordered Probit model," International Journal of Environmental Research and Public Health, vol. 16, no. 14, p. 2632, 2019.

[13] D. J. Sun and L. Elefteriadou, "Lane-changing behavior on urban streets: an "In-Vehicle" field experiment-based study," Computer-Aided Civil and Infrastructure Engineering, vol. 27, no. 7, pp. 525-542, 2012.

[14] P. G. Gipps, "A model for the structure of lane-changing decisions," Transportation Research Part B: Methodological, vol. 20, no. 5, pp. 403-414, 1986.

[15] K. Nagel and M. Schreckenberg, "A cellular automaton model for freeway traffic," Journal de Physique I, vol. 2, no. 12, pp. 2221-2229, 1992.

[16] K. Nagel, D. E. Wolf, P. Wagner, and P. Simon, "Two-lane traffic rules for cellular automata: a systematic approach," Physical Review E, vol. 58, no. 2, pp. 1425-1437, 1998.

[17] P. Hidas, "Modelling vehicle interactions in microscopic simulation of merging and weaving," Transportation Research Part C: Emerging Technologies, vol. 13, no. 1, pp. 37-62, 2005.

[18] H. Yu, H. E. Tseng, and R. Langari, "A human-like game theory-based controller for automatic lane changing," Transportation Research Part C: Emerging Technologies, vol. 88, pp. 140-158, 2018.

[19] Q. Yang and H. N. Koutsopoulos, “A Microscopic Traffic Simulator for evaluation of dynamic traffic management systems," Transportation Research Part C: Emerging Technologies, vol. 4, no. 3, pp. 113-129, 1996.

[20] T. Toledo, H. N. Koutsopoulos, and M. Ben-Akiva, "Integrated driving behavior modeling," Transportation Research Part C: Emerging Technologies, vol. 15, no. 2, pp. 96-112, 2007.

[21] J. Nilsson, J. Silvlin, M. Brannstrom, E. Coelingh, and J. Fredriksson, "If, when, and how to perform lane change maneuvers on highways," IEEE Intelligent Transportation Systems Magazine, vol. 8, no. 4, pp. 68-78, 2016.

[22] S. Das and B. Ba, "Simulations of highway chaos using fuzzy logic," in 18th International Conference of the North American Fuzzy Information Processing Society - NAFIPS (Cat. No.99TH8397), June 1999.

[23] J. Peng, Y. Guo, R. Fu, W. Yuan, and C. Wang, "Multi-parameter prediction of drivers' lane-changing behaviour with neural network model," Applied Ergonomics, vol. 50, pp. 207-217, 2015.

[24] Yi Hou, E. Praveen, and C. Sun, "Modeling mandatory lane changing using Bayes classifier and decision trees," IEEE Transactions on Intelligent Transportation Systems, vol. 15, no. 2, pp. 647-655, 2014.

[25] J. C. McCall, D. P. Wipf, M. M. Trivedi, and B. D. Rao, "Lane change intent analysis using robust operators and sparse bayesian learning," IEEE Transactions on Intelligent Transportation Systems, vol. 8, no. 3, pp. 431-440, 2007.

[26] D. C. K. Ngai and N. H. C. Yung, "A multiple-goal reinforcement learning method for complex vehicle overtaking maneuvers," IEEE Transactions on Intelligent Transportation Systems, vol. 12, no. 2, pp. 509-522, 2011.

[27] V. Butakov and P. Ioannou, "Personalized driver/vehicle lane change models for ADAS," IEEE Transactions on Vehicular Technology, vol. 64, no. 10, pp. 4422-4431, 2015.

[28] M. McNaughton, C. Urmson, J. M. Dolan, and J.-W. Lee in Proceedings of IEEE International Conference on Robotics and Automation, pp. 4889-4895, IEEE, Shanghai, China, May 2011.

[29] A. Halati, H. Lieu, and S. Walker, "CORSIM-corridor traffic simulation model," in Proceedings of Traffic Congestion and Traffic Safety in the 21st Century: Challenges, Innovations, and Opportunities, pp. 570-576, Chicago, IL, USA, June 1997.

[30] M. Rickert, K. Nagel, M. Schreckenberg, and A. Latour, "Two lane traffic simulations using cellular automata," Physica A: Statistical Mechanics and Its Applications, vol. 231, no. 4, pp. 534-550, 1996.

[31] H. Kita, "A merging-giveway interaction model of cars in a merging section: a game theoretic analysis," Transportation Research Part A: Policy and Practice, vol. 33, no. 3-4, pp. 305-312, 1999.

[32] T. Toledo, H. N. Koutsopoulos, and M. Ben-Akiva, "Estimation of an integrated driving behavior model," Transportation Research Part C: Emerging Technologies, vol. 17, no. 4, pp. 365-380, 2009.

[33] https://www.fhwa.dot.gov/publications/research/operations/ 07030/index.cfm. 